\title{
Que peuvent apporter les sciences humaines et sociales à la recherche en médecine d'urgence?
}

\section{How can the Social Sciences and Humanities contribute to emergency medicine research?}

\author{
M. Riou - A. L Feral-Pierssens $\cdot$ C. Tourette-Turgis $\cdot$ K. Tazarourte $\cdot$ Y. Freund $\cdot$ T. Pelaccia $\cdot$ B. Riou \\ (C) SFMU et Lavoisier SAS 2018
}

La recherche française en médecine d'urgence a pris son essor au meilleur niveau international depuis de nombreuses années en ce qui concerne les urgences préhospitalières [1,2] et plus récemment en ce qui concerne les urgences hospitalières [3]. De nombreuses équipes sont maintenant capables de décrocher des financements nationaux importants dans le cadre du programme hospitalier de recherche clinique (PHRC), même si l'étape suivante, l'obtention de fonds européens (ERC), reste encore à franchir. Toutefois ces recherches s'inscrivent dans un sillon classique de la recherche médicale : biologique fondamentale, translationnelle ou

\section{Riou}

Université Lumière Lyon 2, centre de recherche en terminologie et traduction (CRTT), F-69007 Lyon, France

\section{A. L Feral-Pierssens}

Service des urgences, hôpital européen Georges Pompidou, Assistance Publique-Hôpitaux de Paris (AP-HP),

F-75015 Paris, France

\section{Tourette-Turgis}

Sorbonne Université, université des patients, chaire humanité et santé, EA 7529,

Conservatoire national des arts et métiers (Cnam),

F-75013 Paris, France

\section{K. Tazarourte}

Université Claude Bernard Lyon 1, HESPER EA 7425

et département de médecine d'urgence,

groupe hospitalier Edouard Herriot, hospices civils de Lyon, F-69008 Lyon, France

\section{Y. Freund $\cdot$ B. Riou $(\bowtie)$}

Sorbonne Université, service d'accueil des urgences, groupe hospitalier Pitié-Salpêtrière,

Assistance Publique-Hôpitaux de Paris (AP-HP),

47-83 boulevard de l'Hôpital, F-75651 Paris cedex 13, France

e-mail : bruno.riou@aphp.fr

\section{T. Pelaccia}

SAMU 67, hôpitaux universitaires de Strasbourg, université de Strasbourg, faculté de médecine, centre de formation et de recherche en pédagogie des sciences de la santé, F-67000 Strasbourg, France clinique. Or des espaces originaux de recherche s'ouvrent dans le cadre des sciences humaines et sociales (SHS), jusqu'ici peu explorés, alors que des avancées importantes et des retombées rapides pour les patients peuvent être attendues. Lors du dernier Forum de l'urgence qui s'est tenu à Lyon du 5 au 6 décembre 2018 une session entière a été consacrée à cette question : Que peuvent apporter les sciences humaines et sociales à la recherche en médecine d'urgence? Cet éditorial se propose d'en synthétiser les principaux éléments afin d'inciter les équipes d'urgence à nouer des coopérations de recherche fructueuses avec des équipes de recherche en SHS.

\section{Linguistique et régulation médicale}

Les études sur la communication en contexte médical sont de plus en plus nombreuses, s'intéressant par exemple à la consultation chez le médecin généraliste ou au dialogue avec les familles en milieu hospitalier. Peu de travaux ont porté sur la régulation médicale et, pourtant, le seul canal par lequel l'assistant de régulation et le médecin régulateur ont accès au patient passe par le langage. Si l'objectif d'un appel d'urgence est que l'appelant et le régulateur coopèrent efficacement pour rassembler des informations (exemple : le patient respire-t-il ?) et effectuer certaines actions (exemple : réanimation cardio-pulmonaire), il est primordial de mieux comprendre les facteurs langagiers et communicationnels qui influencent la compréhension, les réponses, et les actions de l'appelant. Des travaux récents suggèrent que le choix des mots prononcés par le régulateur a un impact sur l'efficacité de la description que l'appelant fait de la situation [4], et sur son consentement à effectuer une réanimation cardiopulmonaire sur le patient [5]. Ce champ d'investigation immense commence à être exploré dans un contexte anglophone [6,7], mais tout reste à faire dans un contexte francophone [8]. Pour pallier à ce manque de connaissances actuel, il nous faut envisager l'association entre spécialistes de médecine d'urgence et spécialistes de la langue. C'est par ce travail 
interdisciplinaire que l'on pourra poser des questions de recherche directement applicables à la réalité de la régulation médicale, et y répondre grâce une méthodologie adaptée à l'étude de la parole spontanée.

\section{Géographie et médecine d'urgence}

La géographie de la santé a pour objet l'analyse spatiale des inégalités de santé des populations, de leurs comportements et des facteurs de leur environnement qui concourent à l'impact sur leur santé. En médecine d'urgence, l'identification de populations ou de territoires à risque de morbimortalité élevé et l'évaluation de l'accessibilité aux soins urgents (pré et intrahospitalier) sont les éléments pivots pour optimiser l'organisation de prise en charge des patients. L'identification de territoires où l'incidence de survenue d'évènements critiques (arrêt cardiaque, traumatisme grave, accident vasculaire cérébral) est plus élevée qu'ailleurs permet de mieux cibler la prévention et l'implantation de dispositifs de secours [9]. Les recherches sur l'accessibilité aux soins urgents sont récentes en France et, en proposant des outils d'évaluation, ouvrent des perspectives de réorganisation de l'offre de soins et des modalités de prise en charge pour des pathologies où le facteur temps est crucial [10].

\section{Le patient expert}

L'essor des maladies chroniques, le vieillissement de la population, les mutations des comportements de santé modifient la dynamique de la relation et des procédures de soin. Ces changements ont pour effet la délégation aux patients d'un ensemble de tâches d'auto-soin, d'auto-surveillance, et d'autogouvernance de soi, à des fins de santé, de soin et de survivabilité [11]. Ces nouvelles missions léguées aux patients sont encadrées par un ensemble de dispositifs législatifs et réglementaires et de recommandations officielles (Loi hôpital Patients Santé Territoire, Plan cancer, Loi de modernisation de santé de 2016, Ma santé 2022) les désignant comme patients intervenants en éducation thérapeutique, patients experts, patients partenaires, médiateurs pairs en santé mentale, patients enseignants. L'essor de la participation des patients à l'amélioration de l'organisation des soins concerne aussi les services d'urgence. Les recherches prenant en compte l'expérience patient montrent que les patients ont une claire perception des problèmes de communication et de coordination entre les soignants intervenant aux urgences. Ils font des observations pertinentes de climat de travail et cela a des effets de réassurance ou d'inquiétudes [12]. Une étude illustre les 6 comportements de communication ayant un impact direct sur le degré de satisfaction des patients dans les services d'urgence. Ces comportements de communica- tion comportent trois gestes non verbaux comme sourire, serrer la main et produire au moins un geste non médical ayant trait au confort du patient comme arranger son oreiller. Les trois autres comportements verbaux consistent à reconnaître les délais d'attente et s'en excuser, à commencer le dialogue avec une question ouverte du type «comment puis-je vous aider? » et surestimer systématiquement le temps au lieu de le nier et le sous-estimer en croyant bien faire [13]. Il devient de plus en plus pertinent d'intégrer des patients aux urgences en leur confiant des fonctions de patient-partenaire, patient GPS, patient-référent. Ces patients devront bénéficier d'une formation agréée et certifiée de 40 heures afin d'exercer leurs missions en respectant les conditions d'exercice dans un cadre médico-légal. Il existe des outils comme les échelles de mesure du degré de résilience tout à fait adaptés à leur accueil dans un service d'urgence [14].

\section{Sciences sociales et médecine d'urgence}

Alors que le paradigme historique, simpliste, attribuait l'altération de l'état de santé d'un individu à la seule modification de son patrimoine biologique, l'évolution de la médecine a mis en évidence d'autres facteurs tels que les déterminants génétiques ou environnementaux. Le développement des sciences sociales et de leurs champs de recherche a permis d'élaborer un modèle d'analyse encore plus complexe. Celui-ci prend ainsi en compte des paramètres individuels tels que le genre, la situation économique ou le capital social ou culturel ainsi que des paramètres structurels qui touchent à l'organisation des systèmes de santé (déterminants du recours aux soins, modalités de protection sociale, effets des politiques publiques). Les travaux ainsi rendus possibles s'élaborent grâce à la coopération fructueuse de médecins, de sociologues, d'économistes, de politistes, d'anthropologues ou d'épidémiologistes et s'appliquent désormais à la médecine d'urgence.

C'est ainsi que la prise en compte des déterminants socioéconomiques dans la survenue et la prise en charge des pathologies médicales urgentes est devenue incontournable $[15,16]$. Les liens observés entre mortalité et précarité sont le fruit de travaux d'épidémiologie sociale [17] ou d'économie de la santé [18], alimentant le constat d'inégalités sociales de santé en médecine d'urgence. Par ailleurs, dans un contexte de surcharge des services d'urgence et de réorganisation permanente du système de soins primaires, l'analyse des parcours de soins des patients qui aboutissent aux urgences est un axe de recherche crucial qu'il apparait indispensable d'explorer. Cette analyse ne peut pas faire l'économie des approches ethnographiques ou anthropologiques, ces dernières portant sur l'expérience du corps et de la maladie, sur la perception de l'urgence (réelle ou ressentie) ou sur l'analyse des interactions entre patients et soignants [19]. À celles-ci 
s'ajoutent les approches des sociologues et politistes qui interrogent quant à eux, l'organisation, la connaissance, le recours et l'utilisation des systèmes de santé [20].

La contribution des sciences sociales enrichit la recherche en médecine d'urgence tant par les sujets qu'elles interrogent - l'urgence vitale, l'urgence sociale, la singularité des individus ou la construction sociale et politique des soins d'urgence - que par les méthodes quantitatives et qualitatives qu'elles emploient. Ainsi, la synergie entre ces différentes disciplines permet à notre spécialité de sortir des sentiers battus et par les propositions qui en découlent, de contribuer à l'amélioration de l'état de santé des populations.

\section{Le raisonnement diagnostique en médecine d'urgence}

Le diagnostic est au cœur de l'activité des médecins urgentistes. La médecine d'urgence est en effet considérée comme l'une des spécialités dont la densité diagnostique et le taux d'erreurs diagnostiques sont les plus élevés. Depuis la fin des années soixante-dix, l'activité diagnostique des médecins est étudiée sous l'angle de la psychologie cognitive, afin d'identifier les processus de raisonnement mobilisés pour diagnostiquer et l'influence de l'environnement sur la prise de décisions. Les recherches réalisées spécifiquement sur le raisonnement clinique en médecine d'urgence sont rares. Elles ont notamment conduit à révéler le rôle central de l'intuition, qui permet aux médecins urgentistes de générer la plupart des hypothèses avant la rencontre avec le patient ou dans les cinq premières minutes de cette rencontre [21]. Elles ont également révélé l'usage, par les experts, d'un type particulier de connaissances, dénommées KnowWho, qui désigne les connaissances qu'ont les médecins urgentistes à propos des personnes avec lesquelles ils travaillent [22]. Ces connaissances leur servent en particulier à filtrer les informations communiquées par un tiers, en leur accordant du poids ou, au contraire, en les relativisant. La production de savoirs, pour le moment embryonnaires, dans le domaine du raisonnement clinique appliqué à la médecine d'urgence est associée à des enjeux majeurs sur le plan de la qualité des soins, de l'amélioration de la performance diagnostique et de la réduction des erreurs.

\section{Erreurs en médecine d'urgence}

L'étude de la culture de l'erreur et des moyens de s'en prémunir est relativement récente dans le monde médical, et a pris son essor après la publication en $1999 \mathrm{du}$ rapport « To err is human » [23]. En médecine d'urgence, la recherche sur l'erreur médicale débute très tard, et ce n'est qu'après 2010 que les premières études prospectives cherchent à évaluer la prévalence et les facteurs associés à l'erreur aux urgences. Que ce soit aux États-Unis ou en France, une prévalence importante de l'erreur médicale aux urgences est rapportée : près de $10 \%$ des malades seraient victimes d'erreur médicale, associée ou non à un événement indésirable [24]. Le grand Karl Popper le disait déjà en 1960 : si les sources de la connaissance et de l'ignorance sont complexes, celles de l'erreur peuvent être assez triviales [25]. À partir d'une première étude exploratoire, a surgi l'hypothèse que la participation de plus d'un médecin à la prise en charge d'un malade aux urgences (par exemple s'il y a eu transmission) était un facteur protecteur d'erreur. Cette hypothèse s'est vue confirmée indirectement par une étude en réanimation, qui retrouvait une amélioration du pronostic lorsque le médecin de nuit était différent de celui de jour [26]. Les transmissions et la participation de plus d'un médecin à la prise en charge semblent donc être salvateur. Là où l'on craignait auparavant un risque de perte d'informations, on peut à présent réaliser que c'est l'occasion d'un nouveau regard et d'un apport d'information. Être deux à décider, voilà qui pourrait limiter le risque de décisions absurdes, chères au sociologue Christian Morel [27]. Ainsi, l'étude CHARMED a montré une diminution significative du risque d'erreur médicale par l'introduction de vérifications croisées systématiques entre médecin. Ces séances de cross-checking, implémentées toutes les 2-3 heures et d'une durée inférieure à 10 minutes par médecin permettaient de diminuer de $10 \%$ à $6 \%$ le risque d'erreur médicale aux urgences [28].

Ces quelques exemples montrent l'extraordinaire richesse des recherches qui peuvent être conduites dans le cadre de la médecine d'urgence en coopération avec les SHS. La liste n'est pas limitative et nous aurions pu aussi aborder des thématiques comme la télémédecine où l'urgentiste possède une expertise certaine mais sans activité de recherche reconnue par des travaux internationaux, ou la psychologie comportementale, l'anthropologie sociale, la science des organisations, le management. Ceci suppose que se créent des liens entre des urgentistes intéressés par les SHS et des chercheurs en SHS intéressés par la médecine d'urgence, animés par une volonté commune d'explorer des champs peu labourés jusqu'ici de la recherche. En tout cas, lorsque l'on voit la qualité des revues dans lesquelles sont publiés ces travaux, on ne peut que constater l'appétit et l'ouverture des grandes revues médicales pour ces travaux souvent innovateurs.

Conflits d'intérêts : les auteur déclarent ne pas avoir de liens d'intérêts.

\section{Références}

1. Riou B, Barriot P, Rimailho A, Baud FJ (1988) Treatment of severe chloroquine poisoning. N Engl J Med 318:1-6 
2. Jabre P, Belpomme V, Azoulay E, et al (2013) Family presence during cardiopulmonary resuscitation. N Engl J Med 368:1008-18

3. Freund Y, Lemachatti N, Krastinova E, et al (2017) Prognostic accuracy of Sepsis-3 criteria for in-hospital mortality among patients with suspected infection presenting to the emergency department. JAMA 317:301-8

4. Riou M, Ball S, Williams TA, et al (2017) "Tell me exactly what's happened": When linguistic choices affect the efficiency of emergency calls for cardiac arrest. Resuscitation 117: 8-65

5. Riou M, Ball S, Whiteside A, et al (2018) "We're going to do CPR": A linguistic study of the word used to initiate dispatcherassisted CPR and their association with caller agreement. Resuscitation 133:95-100

6. Penn, C, Koole, T, Nattrass, R, et al (2016) When seconds count: A study of communication variables in the opening segment of emergency calls. J Health Psychol 22:1-9

7. Riou M, Ball S, Williams TA, et al (2018) "She's sort of breathing": What linguistic factors determine call-taker recognition of agonal breathing in emergency calls for cardiac arrest. Resuscitation 122:92-8

8. Laforest M, Rioux-Turcotte J (2016) La personne déclarée morte par l'appelant au service téléphonique d'urgence: une source de désalignement interactionnel. Communiquer 18:65-83

9. Castra L, Genin M, Escutnaire J, et al (2019) Socioeconomic status and incidence of cardiac arrest: a spatial approach to social and territorial disparities. Eur J Emerg Med 26:[in press]

10. Freyssenge J, Renard F, Schott AM, et al (2018) Measurement of the potential geographic accessibility from call to definitive care for patient with acute stroke. Int J Health Geogr 17:1

11. Tourette-Turgis C (2015). Éducation thérapeutique du patient : La maladie comme occasion d'apprentissage. Bruxelles, De Boeck

12. Ebadi A, Froutan R, Malekzade J (2019) The design and psychometric evaluation of the emergency medical services resilience scale (EMSRS). Int Emerg Nurs 42:[in press]

13. Finefrock D, Patel S, Zodda D, et al (2018) Patient-centered communication behaviors that correlate with higher patient satisfaction scores. J Patient Exp 5:231-5

14. Henry BW, McCarthy DM, Nannicelli AP, Seivert NP, Vozenilek JA (2016) Patients' views of teamwork in the emergency department offer insights about team performance, Health Expect 19:702-15
15. Feral-Pierssens AL, Aubry A, Truchot J, et al (2016) Emergency care for homeless patients: A French multicentre cohort study. Am J Public Health 106:893-8

16. Bige N, Hejblum G, Baudel JL et al (2015) Homeless patients in the ICU: An observational propensity-matched cohort study. Crit Care Med 43:1246-54

17. Gray AM (1982) Inequalities in health. The Black Report: a summary and comment. Int J Health Serv 12:349-80

18. Chetty R, Stepner M, Abraham S et al (2016) The association between income and life expectancy in the United States, 20012014. JAMA 315:1750-66

19. Vassy C (2004) L'organisation des services d'urgence entre le social et le sanitaire. Mouvement 2:67-74

20. Belorgey N (2010) L'hôpital sous pression. Ed La découverte: Paris

21. Pelaccia T, Tardif J, Triby E, et al (2014) How and when do expert emergency physicians generate and evaluate diagnostic hypotheses? A qualitative study using head-mounted video cued-recall interviews. Ann Emerg Med 64:575-85

22. Pelaccia T, Tardif J, Triby E, et al (2016). From context comes expertise: How do expert emergency physicians use their knowwho to make decisions? Ann Emerg Med 67:747-51

23. Kohn L, Corrigan J, Donaldson M (1999) To err is human: Building a safer health system US Institute of Medicine, Academic press, Washington DC

24. Camargo CA, Tsai CL, Sullivan AF, et al (2012) Safety climate and medical errors in 62 US emergency departments. Ann Emerg Med 60:555-63

25. Popper K (1960) Des sources de la connaissance et de l'ignorance. Réédition 2018, Rivages, Barcelone

26. Kajdacsy-Balla Amaral AC, Barros BS, Barros CC, Innes C, Pinto R, Rubenfeld GD (2014) Nightime cross-coverage is associated with decreased intensive care unit mortality. A singlecenter study. Am J Respir Crit Care Med; 189:1395-401

27. Morel C (2018) Les décisions absurdes III. L'enfer des règles. Les pièges relationnels. Gallimard, Paris

28. Freund Y, Goulet H, Cachanado M, et al (2018) Systematic physician cross-checks to reduce adverse events in the emergency department: The CHARMED cluster randomized trial. JAMA Intern Med 178:812-9 